\title{
Changes in Plasma Levels of FSH, LH and Testosterone during Anti-androgenic Hormone Therapy for Patients with Prostatic Cancer
}

\author{
Masafumi Shirai, Shotaro Matsuda, Shiro Mrtsukawa, \\ Mamoru Nakamura* and Kenzo Yonezawa $\dagger$ \\ Department of Urology, Department of Radiology, ${ }^{*}$ and Hospital \\ Pharmacy, $\dagger$ Tohoku University School of Medicine, Sendai
}

\begin{abstract}
Shirai, M., Matsuda, S., Mitsukawa, S., Nakamura, M. and Yonezawa, K. Changes in Plasma Levels of FSH, LH and Testosterone during Anti-androgenic Hormone Therapy for Patients with Prostatic Cancer. Tohoku J. exp. Med., 1974, 114 (2), 145-158 — Plasma levels of FSH, LH and testosterone were determined in 13 patients of ages from 44 to 85 who had been orchidectomized on account of prostatic cancer and most of whom were receiving additional treatments with anti-androgenic hormone. In 12 of them a LH-RH loading test was performed to examine its effects on the pituitary secretory function and on the adrenal cortical function of testosterone secretion. In 1 of the 12 cases a HCG loading test was also applied to study response of the adrenal gland to HCG. It was found that the combined therapy of orchidectomy with subsequent administration of anti-androgenic hormone caused a rapid fall of the plasma levels of FSH, LH and testosterone, whereas in the early stage the pituitary continued to respond to LH-RH loading. Clinically, pain in urination and that resulted from bone metastases thoroughly disappeared after this combined therapy, although no appreciable changes were seen in the prostatic tumor itself. After more than 6 months' application of anti-androgenic hormones, the plasma level of testosterone began to rise. However, there was no correlation between the increase in plasma testosterone and the aggravation of the tumor. _—anti-androgenic hormone; FSH; LH; prostatic cancer; testosterone
\end{abstract}

Since Huggins and Hodges (194I) suggested the connection between prostatic cancer and androgen, a variety of anti-androgenic hormone therapies have been applied to treat the patients with prostatic cancer. We have also been making use of anti-androgenic hormone therapy in treating patients with prostatic cancer following an orchidectomy or an additional total prostatectomy when such operation was possible. In cases of advanced prostatic cancer, such operative and hormonal treatments may further be combined with surgical removal of the adrenal gland, medical adrenalectomy and intrapituitary irradiation with a radon needle. In addition, lateral chordotomy is also applied to some cases suffering from severe pain caused by bone metastases or other complications.

Thus, irrespective of varying clinical features, we routinely combine the antiandrogenic hormone therapy with preceding surgical procedures for treatment of patients with prostatic cancer. Recently, however, growing doubts have been

Received for publication, August 27, 1974. 
cast upon the effects of anti-androgenic hormone therapy, particularly on the issuing of long-term massive doses of estrogen.

The present study was designed to try and clarify whether or not the longterm massive dosing hormone therapy is actually effective in the treatment for cancer of the prostate. In this series of experiments with prostatic cancer patients, investigations were conducted to identify responses to estrogen administration at varying stages, from the beginning of treatment through the long-term massive dosing. Plasma levels of FSH, LH and testosterone were determined in the patients, and with further loading of luteinizing hormone-releasing hormone (LH$\mathrm{RH}$ ), the changes in these hormone levels were studied to evaluate the effects of anti-androgenic hormone administration in suppressing pituitary function. The relationship between the determined changes in levels of these hormones and the clinical symptom was also discussed.

\section{Materials and Methods}

The subjects were 13 patients chosen at random from the patients with prostatic cancer seen at the Department of Urology, Tohoku University Hospital (Table 1). These subjects, ranging in age from 44 to 85 years, were all confirmed through a biopsy of prostate tissue as having prostatic cancer. Of the 13 patients, 10 already had cancer infiltration in the surrounding tissues and/or bone metastases, thus making a total prostatectomy impossible. These 10 cases were subjected, therefore, to only orchidectomy and subsequent anti-androgenic hormone administration. Among the 10, 1 patient (Case 6) had advanced bone metastases with severe pains at the loins and femoral region, and underwent additional medical adrenalectomy with an adrenocortical hormone. The remaining 3 patients underwent orchidectomy and additional surgery; total prostatectomy in 2 and transurethral prostatectomy in 1 , followed by anti-androgenic hormone administration. The anti-androgenic hormones and adrenocortical hormone used in this series are shown in Table 1, which also includes the doses and the periods applied.

In 3 of the 13 cases (Cases 1, 2 and 3), plasma levels of FSH, LH and testosterone were measured prior to the orchidectomy, and changes in the levels of these hormones were studied at various stages of the post-castration administration of anti-androgenic hormones. In 12 cases, LH-RH loading tests were performed; plasma levels of FSH, LH and testosterone were first determined for the fasting blood sampled in the morning, then an intramuscular injection of $0.1 \mathrm{mg}$ of synthesized $\mathrm{LH}-\mathrm{RH}$ was given to the patients and changes in these hormone values were examined for the blood samples taken at 30,60,90 and $120 \mathrm{~min}$ after injection. In 1 case (Case 11 ), a HCG loading test was carried out to study changes in ability of the adrenal gland in testosterone secretion, namely, HCG (5000 IU) was given once daily for 2 days, and the changes in plasma testosterone levels were evaluated. For the determination of the hormone levels the following two radioimmunoassay methods were utilized: the one by Yonezawa et al. (1973) for FSH and LH, and the other by Shirai et al. (1974) for testosterone.

\section{Results}

1. Changes in plasma levels of FSH, LH and testosterone over a period of time in cases being administered with anti-androgenic hormones following orchidectomy

In 3 of the orchidectomized patients who were subjected to investigation, 2 (Cases 2 and 3 ) showed somewhat higher levels of FSH and LH and lower levels 
of testosterone than the normal levels prior to the operation, while in the other 1 (Case 1) these levels were all normal as shown in Table 2 (normal ranges in males: 1.9-20.0 mIU/ml for FSH, 1.5-19.9 $\mathrm{mIU} / \mathrm{ml}$ for $\mathrm{LH}$, and $430-1180 \mathrm{ng} / 100 \mathrm{ml}$ for testosterone). Plasma levels of these hormones measured in Case 3 showed that 3 days after orchidectomy, plasma testosterone markedly decreased, whereas the levels of both FSH and LH increased prominently, indicating a lessening of the suppression of secretory function of the pituitary gland. After 6 to 8 days, plasma gonadotropin began to decrease, while the testosterone level was still decreasing and approaching the normal female range (34-38 ng/100 ml). Furthermore, after about 21 days, suppression of the secretion of gonadotropin was more marked and the level of plasma testosterone tended to fall below the female range.

\section{Changes in plasma levels of FSH, LH and testosterone by LH-RH loading (Table 3)}

i) Changes in plasma levels of FSH, LH and testosterone by LH-RH loading in a case having undergone only orchidectomy (Fig. 1)

An LH-RH loading test was performed on Case 13, which had been treated only by orchidectomy. Prior to the test, the patient had excessively high levels of plasma FSH $(120.0 \mathrm{mIU} / \mathrm{ml}), \mathrm{LH}(92.0 \mathrm{mIU} / \mathrm{ml})$, and also of testosterone $(104.0$ $\mathrm{ng} / 100 \mathrm{ml}$, three times as high as the normal female level). LH-RH loading caused a marked rise in both plasma FSH and LH levels, showing the peaks appearing at $60 \mathrm{~min}$ and increase-rates of 1.6 times for FSH and 3.6 times for LH. On the other hand, the level of plasma testosterone reached a peak at $30 \mathrm{~min}, 1.2$ times higher than the control levels. The level then began to decline, eventually falling below the control level. However, the levels were at all times higher than the normal female level; the level remained about 2 times as high even at the lowest point.

ii) Variation of plasma levels of FSH, LH and testosterone with the duration of antiandrogenic hormone administration

Irrespective of the daily doses or varieties of anti-androgenic hormones, the subjects were categorized into 4 groups only according to the duration of antiandrogenic hormone therapy: less than 1 month, 1-6 months, 7-24 months, and more than 24 months groups (Table 4). The average plasma levels of FSH were highest in the group of "less than 1 month," and gradually fell to the lowest level in "7-24 months," then tended to rise again in "more than 24 months." The response to LH-RH loading was positive in all 4 groups. The highest levels of plasma FSH in each of the 4 groups were 1.8, 1.4, 2.0 and 1.1 times, respectively (Table 4, Fig. 2). With respect to the average plasma levels of $\mathrm{LH}$, the changes with the lapse of time were similar to those of FSH. The response to LH-RH loading was most prominent in "less than 1 month" with a peak level of $104.6 \pm$ 91.5 (7.9 times as high as the base line), showing a still insufficient suppression of the pituitary secretory function. The suppressing effect became evident after 6 months, with the subsequent weakening of the response to LH-RH. The differ- 
Table 1. Cases

\begin{tabular}{|c|c|c|c|c|c|c|c|}
\hline \multirow{3}{*}{$\begin{array}{l}\text { Case } \\
\text { No. }\end{array}$} & \multirow{3}{*}{ Name } & \multirow{3}{*}{ Age } & \multirow{3}{*}{$\begin{array}{l}\text { Chief } \\
\text { complaint }\end{array}$} & \multicolumn{4}{|c|}{ Methods of therapy } \\
\hline & & & & \multirow{2}{*}{$\begin{array}{c}\text { Operative } \\
\text { therapy }\end{array}$} & \multicolumn{3}{|c|}{ Hormone therapy } \\
\hline & & & & & $\begin{array}{l}\text { Kind of } \\
\text { hormone }\end{array}$ & $\begin{array}{c}\text { Dosage } \\
\text { (mg/day) }\end{array}$ & Duration \\
\hline 1 & Y.T. & 75 & Pollakisuria & O. & $\mathrm{H}$. & 27 & 13 days \\
\hline 2 & R.S. & 70 & $\begin{array}{l}\text { Dysuria, } \\
\text { lumbago }\end{array}$ & 0 & S. & 250 & 14 days \\
\hline 3 & S.T. & 75 & $\begin{array}{l}\text { Lumbago, } \\
\text { dysuria }\end{array}$ & 0 & H. & 27 & 14 days \\
\hline 4 & T.T. & 75 & $\begin{array}{c}\text { Lumbago, } \\
\text { dysuria }\end{array}$ & 0. & $\begin{array}{l}\text { S. } \\
\text { S. } \\
\text { H. }\end{array}$ & $\begin{array}{r}500 \\
900 \\
27\end{array}$ & $\begin{array}{r}7 \text { days } \\
7 \text { days } \\
14 \text { days }\end{array}$ \\
\hline 5 & H.S. & 60 & Dysuria & 0. & $\begin{array}{l}\text { S. } \\
\text { H. }\end{array}$ & $\begin{array}{r}250 \\
27\end{array}$ & $\begin{array}{l}8 \text { days } \\
5 \text { mo }\end{array}$ \\
\hline 6 & H.O. & 44 & $\begin{array}{l}\text { Lumbago, } \\
\text { paralysis } \\
\text { of rt. leg }\end{array}$ & 0 & $\begin{array}{l}\text { H. } \\
\text { H. } \\
\text { D. }\end{array}$ & $\begin{array}{r}30 \\
45 \\
2\end{array}$ & $\begin{array}{c}4 \text { mo } \\
40 \text { days }\end{array}$ \\
\hline & T.T. & 71 & Dysuria & o. & $\mathrm{H}$. & 27 & $6 \mathrm{mo}$ \\
\hline 8 & T.T. & 67 & Dysuria & O. \& T.T.P. & $\mathbf{H}$. & 27 & $1 \mathrm{yr} 3 \mathrm{mo}$ \\
\hline 9 & F.T. & 59 & $\begin{array}{l}\text { Dysuria, } \\
\text { lumbago }\end{array}$ & 0. & H. & 36 & $1 \mathrm{yr} 8 \mathrm{mo}$ \\
\hline 10 & M.S. & 85 & Pollakisuria & 0 . & $\begin{array}{l}\text { S. } \\
\text { S. }\end{array}$ & $\begin{array}{l}250 \\
600\end{array}$ & $\begin{array}{l}10 \text { days } \\
4 \text { yr } 2 \text { mo }\end{array}$ \\
\hline 11 & K.K. & 76 & Dysuria & O. \& T.T.P. & H. & 27 & $6 \mathrm{yr} 2 \mathrm{mo}$ \\
\hline 12 & T.S. & 70 & Dysuria & $\begin{array}{l}\text { O. \& } \\
\text { T.U.R.Pt. }\end{array}$ & $\begin{array}{l}\text { S. } \\
\text { H. } \\
\text { H. }\end{array}$ & $\begin{array}{r}250 \\
27 \\
45\end{array}$ & $\begin{array}{l}5 \mathrm{mo} \\
2 \mathrm{yr} 3 \mathrm{mo} \\
3 \mathrm{yr} 2 \mathrm{mo}\end{array}$ \\
\hline 13 & Y.I. & 72 & $\begin{array}{l}\text { Feeling of } \\
\text { residual } \\
\text { urine, pol- } \\
\text { lakisuria }\end{array}$ & 0. & & & \\
\hline
\end{tabular}


of prostatic cancer

\begin{tabular}{|c|c|c|c|c|}
\hline \multicolumn{4}{|c|}{ Efficiency of therapy } & \multirow{3}{*}{ Metastasis } \\
\hline \multicolumn{2}{|c|}{ Prostate } & \multicolumn{2}{|c|}{ Urination and others } & \\
\hline Before treatment & $\begin{array}{c}\text { After } \\
\text { treatment }\end{array}$ & Before treatment & $\begin{array}{l}\text { After } \\
\text { treatment }\end{array}$ & \\
\hline $\begin{array}{l}\text { Hen egg size, asym- } \\
\text { metric, uneven, hard }\end{array}$ & No change & Pollakisuria & Cured & $\begin{array}{l}\text { Infiltration to } \\
\text { baldder }\end{array}$ \\
\hline $\begin{array}{l}\text { Over-walnut size, } \\
\text { asymmetric, partially } \\
\text { hard }\end{array}$ & No change & $\begin{array}{l}\text { Dysuria } \\
\text { (retention) }\end{array}$ & $\begin{array}{c}\text { Improved } \\
\text { (R.U. } 35 \mathrm{ml} \text { ) }\end{array}$ & \\
\hline $\begin{array}{l}\text { Over-walnut size, } \\
\text { symmetric, partially } \\
\text { hard }\end{array}$ & No change & Lumbago & Improved & $\stackrel{+}{\text { (bone) }}$ \\
\hline $\begin{array}{l}\text { Hen egg size, asym. } \\
\text { metric, wholly hard }\end{array}$ & Slightly shrunk & Lumbago, dysuria & Improved & $\begin{array}{c}+ \\
\text { (bone) }\end{array}$ \\
\hline $\begin{array}{l}\text { Over-walnut size, } \\
\text { asymmetric, uneven, } \\
\text { partially hard }\end{array}$ & Shrunk & $\begin{array}{c}\text { Dysuria } \\
\text { (R.U. } 50 \mathrm{ml})\end{array}$ & $\begin{array}{c}\text { Improved } \\
\text { (R.U. } 12 \mathrm{ml})\end{array}$ & \\
\hline $\begin{array}{l}\text { Over-walnut size, } \\
\text { asymmetric, wholly } \\
\text { hard }\end{array}$ & Slightly shrunk & $\begin{array}{c}\text { Lumbago, paralysis } \\
\text { of rt. leg }\end{array}$ & $\begin{array}{l}\text { Remarkably } \\
\text { improved }\end{array}$ & $\stackrel{+}{+}$ \\
\hline $\begin{array}{l}\text { Hen egg size, sym- } \\
\text { metric, smooth, } \\
\text { wholly hard }\end{array}$ & Shrunk & $\begin{array}{c}\text { Dysuria } \\
\text { (R.U. } 100 \mathrm{ml} \text { ) }\end{array}$ & $\begin{array}{l}\text { Improved } \\
\text { (R.U. } 8 \mathrm{ml})\end{array}$ & $\begin{array}{c}+ \\
\text { (bone) }\end{array}$ \\
\hline $\begin{array}{l}\text { Over-walnut size, } \\
\text { asymmetric, wholly } \\
\text { hard }\end{array}$ & & Dysuria & Cured & \\
\hline $\begin{array}{l}\text { Under-hen egg size, } \\
\text { nodular, partially } \\
\text { hard }\end{array}$ & $\begin{array}{l}\text { Remarkably } \\
\text { shrunk }\end{array}$ & Dysuria, lumbago & $\begin{array}{l}\text { Improved } \\
\text { Cured }\end{array}$ & $\begin{array}{c}+ \\
\text { (bone) }\end{array}$ \\
\hline $\begin{array}{l}\text { Over-walnut size, } \\
\text { asymmetric, nodular, } \\
\text { wholly hard }\end{array}$ & $\begin{array}{l}\text { Slightly } \\
\text { enlarged }\end{array}$ & Pollakisuria & Cured & \\
\hline $\begin{array}{l}\text { Walnut size, uneven, } \\
\text { wholly hard }\end{array}$ & & Dysuria & Cured & \\
\hline $\begin{array}{l}\text { Over-hen egg size, } \\
\text { asymmetric whollly } \\
\text { hard }\end{array}$ & Slightly shrunk & $\begin{array}{l}\text { Dysuria } \\
\text { (retention) }\end{array}$ & $\begin{array}{l}\text { Improved } \\
\text { (R.U. } 10 \mathrm{ml})\end{array}$ & \\
\hline $\begin{array}{l}\text { Over-walnut size, } \\
\text { symmetric, uneven, } \\
\text { partially hard }\end{array}$ & Enlarged & Pollakisuria & No change & \\
\hline
\end{tabular}

T.T.P., transpubic total prostatectomy; T.U.R.Pt., transurethral prostatectomy. 
TABLE 2. Effects of orchidectomy with anti-androgenic hormone therapy upon the levels of plasma FSH, LH and testosterone in cases of prostatic cancer

\begin{tabular}{|c|c|c|c|c|c|}
\hline $\begin{array}{l}\text { Case } \\
\text { No. }\end{array}$ & $\begin{array}{l}\text { Date of measurement } \\
\text { of plasma hormones }\end{array}$ & $\underset{(\mathrm{mIU} / \mathrm{ml})}{\mathrm{FSH}}$ & $\begin{array}{c}\mathrm{LH} \\
(\mathrm{mIU} / \mathrm{ml})\end{array}$ & $\begin{array}{l}\text { Testosterone } \\
(\mathrm{ng} / 100 \mathrm{ml})\end{array}$ & \\
\hline 1 & $\begin{array}{l}\text { Before orchidectomy } \\
6 \text { days after operation }\end{array}$ & $\begin{array}{r}11.3 \\
5.0\end{array}$ & $\begin{array}{l}18.5 \\
15.5\end{array}$ & $\begin{array}{l}660 \\
31.6\end{array}$ & $\begin{array}{l}\text { Hexestrol }(27 \mathrm{mg} / \text { day }) \\
\text { was administered } \\
\text { from } 2 \text { days after } \\
\text { operation. }\end{array}$ \\
\hline 2 & $\begin{array}{l}\text { Before orchidectomy } \\
6 \text { days after operation }\end{array}$ & $\begin{array}{r}32.0 \\
5.0\end{array}$ & $\begin{array}{l}27.0 \\
15.5\end{array}$ & $\begin{array}{l}210 \\
85.0\end{array}$ & $\begin{array}{l}\text { Stilbestrol }(250 \mathrm{mg} / \\
\text { day) was administer- } \\
\text { ed from } 6 \text { days before } \\
\text { operation. }\end{array}$ \\
\hline 3 & $\begin{array}{l}\text { Before orchidectomy } \\
3 \text { days after operation } \\
8 \text { days after operation } \\
21 \text { days after operation }\end{array}$ & $\begin{array}{r}20.0 \\
44.0 \\
11.0 \\
2.6\end{array}$ & $\begin{array}{r}20.0 \\
46.0 \\
10.5 \\
2.0\end{array}$ & $\begin{array}{l}380 \\
67.6 \\
48.0 \\
31.6\end{array}$ & $\begin{array}{l}\text { Stilbestrol }(500 \mathrm{mg} / \\
\text { day) was administer- } \\
\text { ed from I day after } \\
\text { operation. }\end{array}$ \\
\hline
\end{tabular}

ences between the peak and control levels were also reduced accordingly: 7.9, 2.9, 2.1 and 1.3 times, respectively (Table 4, Fig. 3). In plasma testosterone, the situation was somewhat different. Within the first 6 months of anti-androgenic hormone administration, the plasma levels of testosterone remained lower than the normal female level. However, after 6 months, the level of testosterone began to increase gradually beyond the normal female level in response to continued $\mathrm{LH}-\mathrm{RH}$ loading. This rise in testosterone paralleled that of $\mathrm{LH}$.

iii) Variation of plasma levels of FSH, LH and testosterone in response to the administration of different types of anti-androgenic hormones

The anti-androgenic hormones administered in this series were hexestrol and

TABLE 3. Variation of plasma FSH, LH and testosterone

\begin{tabular}{|c|c|c|c|c|c|c|c|}
\hline \multirow{3}{*}{ Case No. } & \multicolumn{5}{|c|}{$\mathrm{FSH}(\mathrm{mIU} / \mathrm{ml})$} & \multirow{3}{*}{ Base level } & \multirow{3}{*}{$\begin{array}{l}\text { After } \\
30\end{array}$} \\
\hline & \multirow{2}{*}{ Base level } & \multicolumn{4}{|c|}{ After LH-RH loading (min) } & & \\
\hline & & 30 & 60 & 90 & 120 & & \\
\hline 1 & 7.0 & 10.0 & 11.0 & 12.5 & 21.0 & 32.0 & 145.0 \\
\hline 3 & 8.0 & 1.6 & 4.6 & 6.2 & 4.2 & 6.2 & 44.0 \\
\hline 4 & 1.9 & 1.9 & 0.9 & 4.4 & 4.2 & 1. 3 & 16.0 \\
\hline 5 & 5.0 & 6.6 & 5.6 & 8.4 & 5.8 & 9.8 & 15.5 \\
\hline 6 & 6.6 & 3.0 & 4.2 & 5.2 & 7.8 & 5.8 & 22.5 \\
\hline 7 & 1.7 & 1.5 & 1.7 & 5.2 & 1.2 & 6.4 & 26.5 \\
\hline 8 & 3.7 & 2. 7 & 1.5 & 2.7 & 3.9 & 9.0 & 6.4 \\
\hline 9 & 1.4 & 2. 0 & 2.7 & 7.8 & 2.4 & 1.2 & 5.2 \\
\hline 10 & 4.2 & 5.2 & 3.0 & 6.1 & 4.9 & 8.1 & 10.5 \\
\hline 11 & 11.0 & 14.0 & 11.0 & 9.6 & 13.3 & 15.2 & 22.8 \\
\hline 12 & 8.5 & 7.5 & 8.0 & 8.7 & 6.0 & 4. 1 & 2.7 \\
\hline 13 & 120.0 & 129.0 & 200.0 & 165.0 & 118.0 & 92.0 & 285.0 \\
\hline
\end{tabular}

* Undetectably low level. 


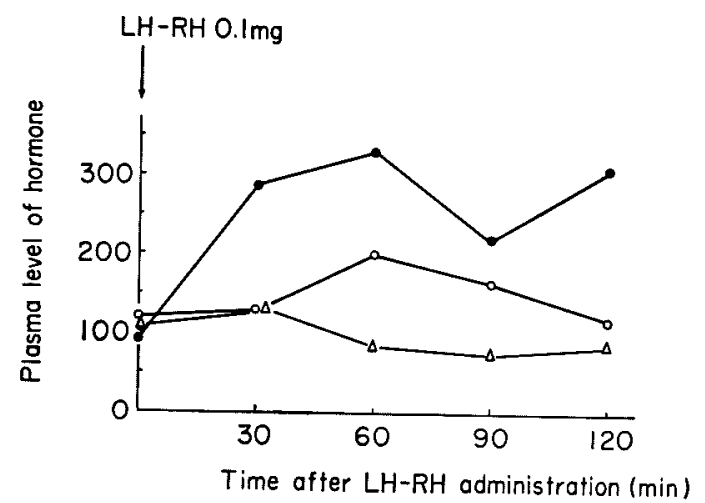

Fig. 1. Variation of levels of plasma FSH, LH and testosterone following LH-RH administration in a patient (Case 13) having undergone only orchidectomy. o, FSH (mIU/ml); •, LH (mIU/ml); $\Delta$, testosterone $(\mathrm{ng} / 100 \mathrm{ml})$.

stilbestrol. It is difficult, however, to compare the therapeutic effects of the two, for in contrast to the relatively long range administration of hexestrol in most cases, the use of stilbestrol was limited to short term massive doses, with a single exception of Case 10. In order to identify any distinctive effects among castrated patients undergoing anti-androgenic hormone treatments which were different in type but identical in duration, 1 patient (Case 6) who received an additional adrenocortical hormone was compared with 2 others (Cases 5 and 7) who received no such additional treatment. Results obtained indicated that in Case 6, the secretion of neither FSH nor LH seemed suppressed and also there was any particularly negative response to LH-RH loading; however, the secretion of testosterone was evidently stopped completely, as there was no response at all to the LH-RH loading.

following $L H \cdot R H$ administration in cases of prostatic cancer

\begin{tabular}{|c|c|c|c|c|c|c|c|}
\hline \multicolumn{3}{|c|}{$\mathrm{LH}(\mathrm{mIU} / \mathrm{ml})$} & \multicolumn{5}{|c|}{ Testosterone $(\mathrm{ng} / 100 \mathrm{ml})$} \\
\hline \multicolumn{3}{|c|}{ LH-RH loading (min) } & \multirow{2}{*}{ Base level } & \multicolumn{4}{|c|}{ After LH-RH loading (min) } \\
\hline 60 & 90 & 120 & & 30 & 60 & 90 & 120 \\
\hline 234.0 & 125.0 & 176.0 & 11.2 & 6.2 & 4.8 & 7.2 & 4.8 \\
\hline 46.0 & 55.0 & 48.0 & 54.0 & 16.8 & 21.6 & 24.0 & 8.4 \\
\hline 34.0 & 31.0 & 54.0 & 34.4 & 32.0 & 28.0 & 16.8 & 16.8 \\
\hline 18.2 & 18.2 & 17.5 & 22.4 & 28.0 & 20.2 & 18,8 & 17.0 \\
\hline 19.0 & 21.0 & 23.5 & $0^{*}$ & 0 & 0 & 0 & 0 \\
\hline 23.0 & 16.5 & 23.0 & 68.0 & 58.0 & 25.8 & 45.8 & 17. 8 \\
\hline 8.0 & 5.7 & 11.5 & 16.8 & 46.0 & 48.0 & 64.0 & 68.0 \\
\hline 8.2 & 11.3 & 10.5 & 55.0 & 60.0 & 40.0 & 26.0 & 61.4 \\
\hline 14.0 & 13.5 & 8.2 & 16.0 & 30.4 & 30.8 & 24.4 & 31.2 \\
\hline 18.0 & 17.5 & 14.0 & 96.0 & 92.0 & 83.0 & 84.0 & 76.0 \\
\hline 2.5 & 5.6 & 2.3 & 92.0 & 96.0 & 100.0 & 93.0 & 104.0 \\
\hline 330.0 & 220.0 & 310.0 & 104.0 & 129.0 & 84.0 & 74.0 & 84.0 \\
\hline
\end{tabular}


TABLE 4. Variation of plasma levels of FSH, $L H$ and testosterone

\begin{tabular}{|c|c|c|c|c|c|c|c|c|}
\hline \multirow{3}{*}{ Duration } & \multicolumn{6}{|c|}{$\mathrm{FSH}(\mathrm{mIU} / \mathrm{ml})$} & \multirow{3}{*}{$\begin{array}{l}\text { Base } \\
\text { level }\end{array}$} & \multirow{3}{*}{$\frac{\text { After }}{30}$} \\
\hline & \multirow{2}{*}{$\begin{array}{l}\text { Base } \\
\text { level }\end{array}$} & \multicolumn{4}{|c|}{ After LH-RH loading (min) } & \multirow{2}{*}{$\mathrm{p} / \mathrm{b}$} & & \\
\hline & & 30 & 60 & 90 & 120 & & & \\
\hline $\begin{array}{l}\text { Shorter than } \\
1 \text { month } \\
\text { ( } 3 \text { cases })\end{array}$ & $5.6 \pm 3.5$ & $4.5 \pm 3.8$ & $5.5 \pm 4.4$ & 7. $7 \pm 3.4$ & $9.8 \pm 7.9$ & 1.8 & $\frac{13.2 \pm}{13.4}$ & $\frac{68.3 \pm}{55.4}$ \\
\hline $\begin{array}{l}1 \sim 6 \\
\text { months } \\
(3 \text { cases })\end{array}$ & $4.4 \pm 2.0$ & $3.7 \pm 2.1$ & $3.8 \pm 1.6$ & $6.3 \pm 1.5$ & $4.9 \pm 2.7$ & 1.4 & $7.3 \pm 1.7$ & $\begin{array}{r}21.5 \pm \\
4.5\end{array}$ \\
\hline $\begin{array}{l}7 \sim 24 \\
\quad \text { months } \\
(2 \text { cases })\end{array}$ & $2.6 \pm 1.1$ & $2.4 \pm 0.3$ & $2.1 \pm 0.6$ & $5.3 \pm 2.5$ & $3.2 \pm 0.7$ & 2.0 & $5,1 \pm 3.9$ & $5.8 \pm 0.6$ \\
\hline $\begin{array}{l}\text { Longer than } \\
24 \text { months } \\
\text { ( } 3 \text { cases) }\end{array}$ & $7.9 \pm 2.8$ & $8.9 \pm 3.7$ & $7.3 \pm 3.3$ & 8. $1 \pm 1.4$ & $8,0 \pm 3.7$ & 1.1 & $9.1 \pm 4.5$ & $\begin{array}{r}12.0 \pm \\
8.2\end{array}$ \\
\hline
\end{tabular}

b, base value; $p$, peak value.

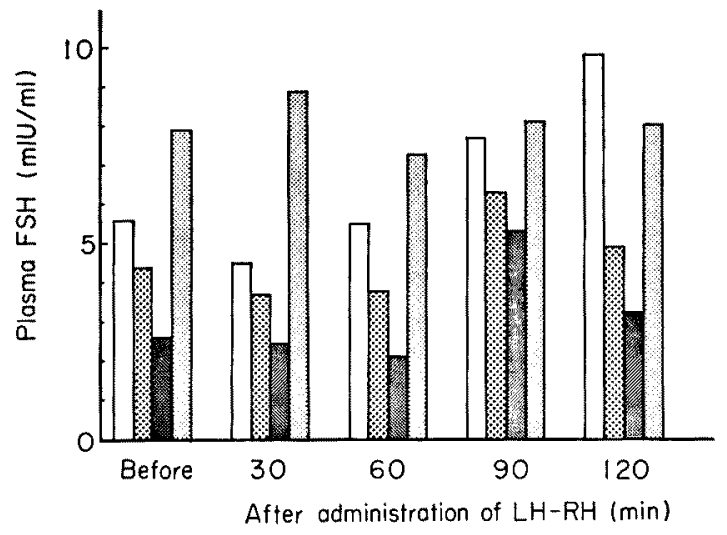

Fig. 2. Variation of plasma levels of FSH with the duration of anti-androgenic hormone administration. $\square$, less than 1 month; $8 \times$, $1 \sim 6$ months; , 7 24 months; more than 24 months.

\section{iv) Variation of plasma levels of FSH, LH and testosterone with the type of operation}

Three of the 13 patients who underwent orchidectomy and an LH-RH loading test underwent additional surgery: total prostatectomy in 2 cases and transurethral prostatectomy in 1 case. However, following these operations, no clear tendencies could be observed in the levels of plasma FSH, LH and testosterone. This leads us to conclude that there is little or no connection between plasma levels of FSH, $\mathrm{LH}$ and testosterone and the types of operation. 
with the duration of anti-androgenic hormone administration

\begin{tabular}{|c|c|c|c|c|c|c|c|c|c|}
\hline \multicolumn{4}{|c|}{$\mathrm{LH}(\mathrm{mIU} / \mathrm{ml})$} & \multicolumn{6}{|c|}{ Testosterone $(\mathrm{ng} / 100 \mathrm{ml})$} \\
\hline \multicolumn{3}{|c|}{ LH-RH loading (min) } & \multirow{2}{*}{$\mathrm{p} / \mathrm{b}$} & \multirow{2}{*}{$\begin{array}{l}\text { Base } \\
\text { level }\end{array}$} & \multicolumn{4}{|c|}{ After LH-RH loading (min) } & \multirow{2}{*}{$\mathrm{p} / \mathrm{b}$} \\
\hline 60 & 90 & 120 & & & 30 & 60 & 90 & 120 & \\
\hline $\begin{array}{r}104.6 \pm \\
91.5\end{array}$ & $\begin{array}{c}70.3 \pm \\
39.8\end{array}$ & $\begin{array}{c}92.7 \pm \\
27.3\end{array}$ & 7.9 & $\begin{array}{l}33.2 \pm \\
17.4\end{array}$ & $\begin{array}{c}18.3 \pm \\
10.5\end{array}$ & $\begin{array}{r}18.1 \pm \\
9.7\end{array}$ & $\begin{array}{r}16.0 \pm \\
6.8\end{array}$ & $\frac{10.0 \pm}{5.0}$ & \\
\hline $\begin{array}{r}20.1 \pm \\
2.1\end{array}$ & $\begin{array}{r}18.6 \pm \\
1.8\end{array}$ & $\begin{array}{r}21.3 \pm \\
2.7\end{array}$ & 2.9 & $\begin{array}{l}30.1 \pm \\
22.3\end{array}$ & $\begin{array}{c}28.7 \pm \\
16.9\end{array}$ & $\begin{array}{r}15.3 \pm \\
6.6\end{array}$ & $\begin{array}{r}21.5 \pm \\
14.1\end{array}$ & $\begin{array}{r}11.6 \pm \\
4.7\end{array}$ & \\
\hline $8.1 \pm 0.1$ & $8.5 \pm 2.8$ & $\begin{array}{r}11.0 \pm \\
0.5\end{array}$ & 2.1 & $\begin{array}{l}35.9 \pm \\
\quad 19.1\end{array}$ & $\frac{53.0 \pm}{7.0}$ & $\begin{array}{r}44.0 \pm \\
\quad 4.0\end{array}$ & $\begin{array}{l}45.0 \pm \\
19.0\end{array}$ & $\begin{array}{r}64.7 \pm \\
\quad 3.3\end{array}$ & 1.8 \\
\hline $\begin{array}{r}11.5 \pm \\
6.5\end{array}$ & $\begin{array}{r}\text { 12. } 2 \pm \\
4.9\end{array}$ & $8.1 \pm 4.7$ & 1.3 & $\begin{array}{r}68.0 \pm \\
36.8\end{array}$ & $\begin{array}{l}72.8 \pm \\
30.0\end{array}$ & $\begin{array}{c}71.2 \pm \\
27.1\end{array}$ & $\begin{array}{c}67.1 \pm \\
30.4\end{array}$ & $\begin{array}{l}70.4 \pm \\
29.0\end{array}$ & 1.1 \\
\hline
\end{tabular}

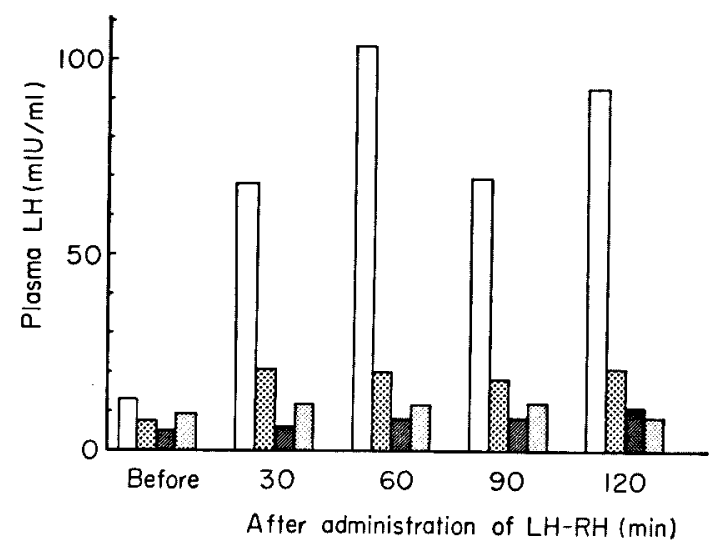

Fig. 3. Variation of plasma levels of $\mathrm{LH}$ with the duration of anti-androgenic hormone administration. $\square$, less than 1 month; $\gg$, 1 6 months; , 7 24 months; 3 , more than 24 months.

\section{Changes in plasma testosterone levels by $H C G$ loading}

The results of the foregoing tests revealed that the plasma level of testosterone tends to rise when the administration of anti-androgenic hormones was continued for more than 6 months.

In response to LH-RH loading, the increasing level of testosterone kept pace with that of LH. To further confirm this trend, a HCG loading test was performed in 1 patient (Case 11) who had received an anti-androgenic hormone consecutively for 6 years after orchidectomy and total prostatectomy. The observed changes 


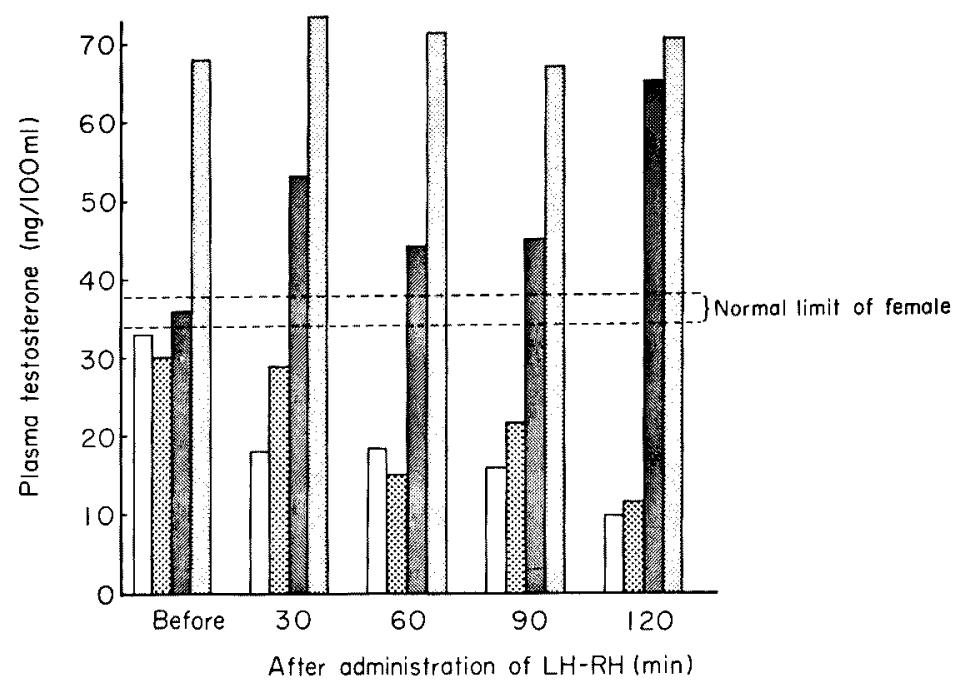

Fig. 4. Variation of plasma levels of testosterone with the duration of anti-androgenic hormone administration. $\square$, less then 1 month; 8 , 1 6 months; 1 , 7 24 months;

in the level of plasma testosterone clearly indicated that HCG raised the plasma testosterone level, showing a peak 1.4 times as high as the control level (Fig. 5).

\section{Relationship between plasma sex hormone levels and clinical symptoms (Table 1)}

In patients who were orchidectomized and subjected to anti-androgenic hormone therapy, suppression of the pituitary secretory function remained insufficient for some time after the start of the therapy, whereas the level of plasma testosterone decreased rapidly. In this early stage of treatment, no appreciable effect on the palpation of the prostatic tumor was apparent, although urination problems were relieved smoothly and there was also considerable relief from the pain caused by bone metastases. With prolonged hormonal therapy, however, suppression of the pituitary secretory function became more evident, in contrast to levels of plasma testosterone which began to increase, and there was a more prominent positive response to LH-RH loading than in the earlier stage of the therapy. In patients having undergone hormonal therapy for less than 2 years, the palpation revealed the shrinkage of prostatic tumor. This contrasted with the situation of patients who, in spite of massive doses of an anti-androgenic hormone for more than 4 years, proved to have a larger tumor than noted before the start of hormonal therapy, though there was no aggravation of urinary conditions.

On the other hand, in Case 13 which had undergone only orchidectomy, there was no evidence of suppressed pituitary secretory function; the levels of plasma FSH, LH and testosterone all markedly increased, the prostatic tumor growing steadily, and troubles with urination remaining without any improvement. 
Case II

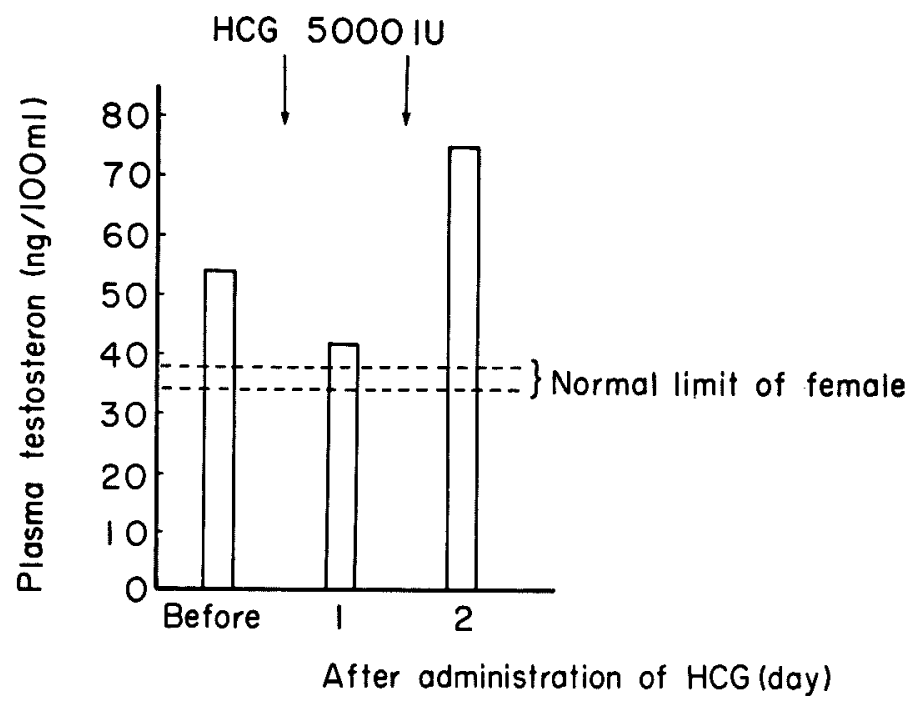

Fig. 5. Response of plasma testosterone to HCG administration in a patient (Case 11) of prostatic cancer.

\section{Discussion}

Since androgen originates in not only the testicle but also the adrenal and ovary, the treatment of prostatic cancer is carried out with an emphasis upon the effective suppression of the secretion of hormones from these organs. Assay of 17-ketosteroid or metabolic products of androgen, which until recently had commonly been employed for estimating the amount of androgen secreted seems now to have given its place to a recently developed direct assay of plasma testosterone. Young and Kent (1968) measured plasma testosterone levels in 3 groups of prostatic cancer patients who had been respectively treated with orchidectomy, with diethylstilbestrol ( $5 \mathrm{mg} / \mathrm{day}$ ), and with a combination of the two. As a result, they found that each treatment caused a marked fall in the level of plasma testosterone, irrespective of the clinical response. Robinson and Thomas (1971) noted that in prostatic cancer patients, the administration of stilbestrol at a daily dose of only $3 \mathrm{mg}$ could reduce the plasma testosterone level to as low as $10 \mathrm{ng} / 100 \mathrm{ml}$ or less, a result which was almost equivalent to the effects of larger dosage, $100 \mathrm{mg}$ per day. Commenting on this conspicuous fall in the level of plasma testosterone, which even dipped below female levels, they reasoned that stilbestrol may directly inhibit the production of androgens in the adrenal cortex, exemplified by the fact that the level of testosterone fell more markedly in orchidectomized patients who underwent additional hormonal therapy with stilbestrol than in those who did not. However, they could not identify a correlation between this drastic fall in testosterone level and any improvement in the clinical conditions of the patients. 
Also the testosterone levels of patients who were orchidectomized and given additional treatments with anti-androgenic hormones were reduced to the female level 6 to 21 days after the start of treatment. At this stage, however, the secretory function of the pituitary was not completely suppressed, and although the levels of plasma FSH and LH were markedly lower than seen before the hormonal therapy, the pituitary gland still responded positively to $\mathrm{LH}-\mathrm{RH}$ loading. It is of interest that with the loading of LH-RH, the level of plasma testosterone fell below the control level and tended to continuously decline with the lapse of time. Although the reason for this tendency is still uncertain, it is evident that at this early stage of treatment, the adrenal cortex does not well respond to LH-RH loading. Clinical conditions arising in relation to the size of the prostate were not significant at this stage, but evidence of early improvement could be seen in both the urination troubles and the pain caused by bone metastases.

Robinson and Thomas (1971), when administering stilbestrol in patients with prostatic cancer, observed that continued dosage for more than 6 months caused the level of plasma testosterone to rise up to between 30 and $80 \mathrm{ng} / 100 \mathrm{ml}$. The interpretation they gave was that the function of the adrenal cortex which had been prevented from secreting testosterone owing to the intervention of stilbestrol probably became released in the course of long continuing dosing, and coupled with an estrogen-induced increase in plasma protein, prompted a protein-testosterone combination which led to a further increase of the bound-type testosterone in plasma. Ochiai (1967), on the other hand, found that long-term massive dosage of estrogen apparently stimulated the adrenal cortex to produce more steroid.

In our experience, it was apparent that a continued administration of antiandrogenic hormones for more than 6 months led to an increase in plasma testosterone. This continued to increase as did the level of plasma LH upon the administration of LH-RH loading. This apparent response of the adrenal cortex to change in plasma LH was further confirmed by another HCG loading test, in which plasma testosterone increased in response to HCG, indicating the sensitivity of the adrenal cortex to LH. These results have led us to believe that the elevation of the level of plasma testosterone with a long-term administration of antiandrogenic hormones is probably attributed to the change in adrenal sensitivity to $\mathrm{LH}$, in addition to a decreased ability of anti-androgenic hormones to suppress androgen synthesis or an increased capacity of these hormones to promote steroid synthesis in the adrenal cortex. In one patient (Case 13) who had undergone only orchidectomy, LH-RH loading caused the plasma sex hormone levels to rise over the control levels, 1.2 times for testosterone, 1.6 times for FSH and 3.6 times for LH, respectively. In such a case, it is presumed that the administration of an anti-androgenic hormone has failed to suppress the synthesis of androgen in the adrenal cortex, and the adrenal sensitivity to gonadotropin has increased, all contributing to the elevation of the level of plasma testosterone.

Robinson and Thomas (1971) applied pituitary ablation with yttrium-90 in prostatic cancer patients suffering from pain of bone metastases, and found that 
within 5 days all the cases showed a reduced plasma testosterone level of below $10 \mathrm{ng} / 100 \mathrm{ml}$, with relief of pain. In another experiment where 11 patients received aminoglutethimide in addition to stilbestrol, 7 showed markedly reduced plasma testosterone levels of less than $10 \mathrm{ng} / 100 \mathrm{ml}$, and in 4 all traces of pain disappeared.

Also in our Case 6 which was suffering from severe pain caused by bone metastases, a combined application of hexestrol and dexamethasone lowered the plasma testosterone concentration to negligible amounts, and the pain disappeared. However, as the response to LH-RH loading was still positive, the administration of dexamethasone was apparently unrelated to the suppression of the function of pituitary to secrete gonadotropin. These findings seem to coincide with those in the previously described experience (Case 6), in which a marked decline in plasma testosterone related to the disappearance of pain even at a stage where the pituitary secretory function was not completely suppressed. This may confirm the existence of a correlation between the low level of plasma testosterone and a shrinkage of the prostatic tumor. Yet it is premature to make such a conclusion, since in some of our cases the long-term administration of hexestrol led to an increase in the level of plasma testosterone, which occurred at the same time as the shrinkage of the tumor. However, if no relationship exists between the boundtype testosterone and either catabolism or androgenic action, as stated by Farnsworth (1970), it is conceivable that the conflicting descriptions above may possibly be due to individual differences, not only in the amount of bound-type testosterone in the increased plasma testosterone, but also in the degree of the sensitivity of the tumor to testosterone.

Comparing prostatic cancer patients in 3 groups which were treated respectively with orchidectomy only, stilbestrol following an orchidectomy, and only stilbestrol without orchidectomy, Young and Kent (1968) noted a marked decrease in plasma testosterone with the lowest mortality rate amongst the patients who underwent only orchidectomy. In our experience, as already been stated, the orchidectomized patient who was given no anti-androgenic hormone showed increased sex hormones in plasma 2 years after the operation, a seizable increase of gonadotropin and moderate one of testosterone, with a positive response to LHRH. The prostatic tumor enlarged somewhat, and there were no visible improvements in such subjective symptoms as dysuria.

Although the number of observable cases are still too few to make any definite conclusions from the test results, they may offer some useful suggestions in regard to the postoperative hormonal therapy for patients with prostatic cancer. Treatments with orchidectomy followed by administration of estrogen usually led to a decrease in plasma testosterone, and in cases where the pituitary showed no response to LH-RH loading, there were no signs of aggravation in the prostatic tumor. This may imply that the therapeutic results of additional treatments with anti-androgenic hormones are more effective than those of orchidectomy alone. Also, the tendency of plasma testosterone levels to rise with long-term 
massive dosage does not necessarily link it to the exacerbation of the prostatic cancer, and there have been no instances of cardiovascular problems such as feared by Robinson and Thomas (1971). The differences between our findings and those of other authors may be attributed to inconsistencies in the chemical structural formulae of stilbestrol used in the experiments, and/or to the racial difference of subjects, though either presumption needs further confirmation.

\section{Acknowledgment}

We gratefully acknowledge Prof. S. Shishito for his instruction throughout this study.

\section{References}

1) Farnsworth, W.E. (1970) The normal prostate and its endocrine control. In: Some Aspects of the Aetiology and Biochemistry of Prostatic Cancer, edited by $\mathrm{K}$. Griffiths \& C.G. Pierrepoint, Alpha Omega Alpha, Cardiff, pp. 3-5.

2) Huggins, C. \& Hodges, C.V. (1941) Studies on prostatic cancer. I. The effects of castration, of estrogen and androgen injection on serum phosphatases in metastatic carcinoma of prostate. Cancer Res., 1, 293-297.

3) Ochiai, K. (1967) Effects of endogenous and exogenous estrogens in man. Folia endocr. jap. (Jap.), 43, 449-453.

4) Robinson, M.R.G. \& Thomas, B.S. (1971) Effects of hormonal therapy on plasma testosterone levels in prostatic carcinoma. Brit. med. J., 4, 391-394.

5) Shirai, M., Matsuda, S., Nakamura, M. \& Yonezawa, K. (1974) A simplified radioimmunoassay for plasma testosterone. Jap. J. clin. Urol. (Jap.), 28, 269-273.

6) Yonezawa, K., Nakamura, M., Shirai, M. \& Matsuda, S. (1973) Measurement of plasma FSH and LH in male gonadal dysfunction with use of HFSH and HLH radioimmunoassay kit. Acta Urol. Jap. (Jap.), 19, 611-616.

7) Young, H.H. \& Kent, J.R. (1968) Plasma testosterone levels in patients with prostatic carcinoma before and after treatment. J. Urol., 99, 788-792. 